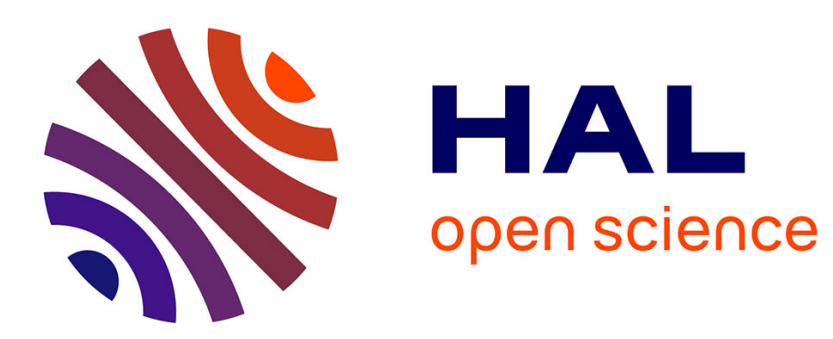

\title{
Timing of grip and goal activation during action perception: a priming study \\ Jérémy Decroix, Solène Kalénine
}

\section{To cite this version:}

Jérémy Decroix, Solène Kalénine. Timing of grip and goal activation during action perception: a priming study. Experimental Brain Research, 2018, 236 (8), pp.2411-2426. 10.1007/s00221-018-53090 . hal-02109222

\section{HAL Id: hal-02109222 \\ https://hal.science/hal-02109222}

Submitted on 5 Jan 2022

HAL is a multi-disciplinary open access archive for the deposit and dissemination of scientific research documents, whether they are published or not. The documents may come from teaching and research institutions in France or abroad, or from public or private research centers.
L'archive ouverte pluridisciplinaire HAL, est destinée au dépôt et à la diffusion de documents scientifiques de niveau recherche, publiés ou non, émanant des établissements d'enseignement et de recherche français ou étrangers, des laboratoires publics ou privés. 
1 Title : Timing of grip and goal activation during action perception : A priming study

2 Author names : Jérémy Decroix \& Solène Kalénine

3 Affiliations : Univ. Lille, CNRS, CHU Lille, UMR 9193 - SCALab - Sciences Cognitives et Sciences

4 Affectives, F-59000 Lille, France

5 Authors' addresse :

6 Laboratoire SCALab UMR CNRS 9193

7 Université de Lille, SHS

8 Batiment A2 - Niveau forum +2

9 Domaine universitaire du Pont de Bois, BP 149

1059653 Villeneuve d'Ascq Cedex, France

Corresponding author: Solène Kalénine

Mail : solene.kalenine@univ-lille3.fr

Phone : +33 320417325

\section{Abstract}

Several models of action recognition acknowledge the involvement of distinct grip and goal representations in the processing of others' actions. Yet their functional role and temporal organisation are still debated. The present priming study aimed at evaluating the relative timing of grip and goal activation during the processing of photographs of object-directed actions. Action could be correct or incorrect owing to grip and/or goal violations. Twenty-eight (Experiment 1) and twenty-five (Experiment 2) healthy adults judged the correctness of target actions according to object typical use. Target pictures were primed by action pictures sharing the same grip or same goal, both the same grip and same goal or none. Primes were presented for 66 or 300ms in Experiment 1 and for 120 or 220ms in Experiment 2. In Experiment 1, facilitative priming effects were observed for goal and grip similarity after $300 \mathrm{~ms}$ primes but only for goal after $66 \mathrm{~ms}$ primes. In Experiment 2, facilitative priming effects were found for both goal and grip similarity from $120 \mathrm{~ms}$ of prime processing. In addition, results from a control condition in Experiment 2 indicated that mere object priming could partially account for goal similarity priming effects, suggesting that object identity may help the observer to make predictions about possible action goals. Findings demonstrate an early and first activation of goal representations, as compared to grip representations, in action decoding, consistent with predictive accounts of action understanding. Future studies should determine to what extent the timing of grip and goal activation is context-sensitive.

Key-words : Action understanding; Goals; Grip; Action semantics; Priming

Decroix, J. \& Kalénine, S. (2018). Timing of grip and goal activation during action perception: A priming study. 
When carrying out actions, humans not only execute movements but aim at achieving goals (Jacob and Jeannerod 2005; Kilner 2011). Yet action goals may be conceptualized at different levels of generality, and action planning and control may occur at multiple levels in the action goal hierarchy. For example, one may reach and grasp a cup (direct goal) to drink or to move it away (distant goal). There is considerable evidence supporting the existence of action planning at these different levels. For instance, performing the same reach and grasp action (direct goal), varies depending on the next action step (distant goals, Ansuini, Santello, Massaccesi, \& Castiello, 2005; Quesque, Lewkowicz, Delevoye-Turrell, \& Coello, 2013). In addition, the neural mechanisms underlying direct and distant goals can be dissociated during action planning (van Schie \& Bekkering, 2007). Overall, the action planning literature highlights the importance of distant goals (such as action intentions or outcomes) and direct goals (such as kinematics parameters, e.g. reach trajectory, grip configuration; Grafton \& Hamilton, 2007) in guiding object-directed actions (Thill et al. 2013; Cooper et al. 2014; van Elk et al. 2014).

In line with the claim of close action-perception relationships (Barsalou 2008; Gentsch et al. 2016), the ability to understand observed actions have been proposed to involve a similar structure as the one identified during action planning (Wolpert et al. 2003; Grafton and Hamilton 2007). Several findings indicate that humans are specifically tuned to interpret others' movements as intended and goal-directed (Hrkać et al. 2014; Novack et al. 2016). Consistent with this idea, fMRI and TMS studies consistently report brain dissociations between the regions involved in decoding action kinematic parameters (reach, grip, effector) and those associated with processing goals (Grafton and Hamilton 2007; Cattaneo et al. 2010; Jacquet and Avenanti 2015; Wurm and Lingnau 2015). Yet the functional role and the temporal organization of kinematics ("how") and goal ("what for") representations during decoding of observed actions remain a matter of debate.

On the one hand, "bottom-up" approaches consider action decoding as bottom-up propagation from the processing of kinematic parameters to the activation of goal representations (Zentgraf et al. 2011; Rizzolatti and Fogassi 2014). Consistent with these views, several studies have demonstrated that there is sufficient invariant information in movements kinematics to discriminate between different action goals (e.g., grasp to pour vs. grasp to drink) and that this information is indeed used by the observers to anticipate the goal of the observed action (Ansuini et al. 2014; Cavallo et al. 2016). On the other hand, "top-down" approaches suggest that contextual information is first used to compute a prediction about the forthcoming action, the actual kinematics being then used to evaluate this prediction (Kilner et al. 2007; Bach et al. 2014). Visual context is indeed known to influence action decoding (Wurm and Schubotz 2012, 2016) and recent evidence suggests that prior knowledge about others' goals directly biases processing of kinematic parameters (Hudson et al. 2016a, b).

Together, bottom-up and top-down approaches are difficult to disentangle based on empirical evidence. Processing of action kinematics contributes to subsequent decisions about action intentions, but it does not inform about whether movement analysis is driven by the prior activation of a representation of the possible action goal. Similarly, although contextual information influences the processing of observed actions, it remains to clarify whether predictions about possible action goals guide action decoding in the absence of externally-driven expectancies (visual scene, prior knowledge about other's intentions). The present study aims at separating bottomup and top-down approaches of action understanding based on temporal data, as suggested by Catmur (2015). Indeed, whereas bottom-up approaches imply that representations of action kinematics would be first activated, top-down approaches suggest that goal representations would be first recruited. 
Assessing the timing of grip and goal activations during action perceptual processing requires to experimentally manipulate information about action kinematics and action goal independently. A few behavioural experiments have dissociated kinematic and goal information during the processing of visual actions (van Elk et al. 2008; Kalénine et al. 2013). Kalénine et al. (2013) found that healthy participants were slower to determine that two videos displayed different actions when the two actions differed only in terms of kinematic parameters (e.g. applying detergent with circular versus straight wipe), or in terms of goals (e.g., applying versus removing detergent), as compared to when the two actions differed in terms of both kinematic and goal. Van Elk et al. (2008) showed that response times to determine that the goal of the action displayed in a picture was correct ("Is the object held at the correct goal location?" e.g., cup near the ear versus near the mouth) were slower when the grip component of the action was incorrect. Conversely, response times to decide whether the grip was correct (i.e., "Is the object grasped with an appropriate grip?" e.g., pencil grasped with power clench versus precision grip) were slower when the goal was incorrect. Moreover, interference from incorrect goals was greater than from incorrect grips. Overall, both studies support the hypothesis that action representations at the kinematic and goal levels play distinct but complementary roles in the processing of observed actions. However, although they provide empirical evidence about the relative "weight" of the different action dimensions in decisions about perceived actions, previous studies do not directly inform about the timing of processing of grip and goal information during action decoding.

Using a priming paradigm involving action pictures containing action violations, the present study goes one step further by investigating the relative timing of grip and goal activation during action recognition. Target actions were judged correct or incorrect after being primed by action pictures sharing the same grip and/or the same goal. The manipulation of different prime durations allowed to directly assess the timing of activation of grip and goal action representations. Prime durations were designed according to EEG studies on perceptual processing of object-directed actions. In these studies, brain activity of participants is recorded while they are determining the goal of an observed action. Overall, decoding others' action goals impacts brain activity in different time windows, the earliest starting around $60 \mathrm{~ms}$ (Ortigue et al. 2009) and the latest emerging from $300 \mathrm{~ms}$ (Ortigue et al. 2009; van Elk et al. 2012; Avanzini et al. 2013) of visual action processing. Thus, 60 and $300 \mathrm{~ms}$ were chosen as prime duration boundaries. The rationale was that short prime durations should only allow the processing of the first steps of action decoding. Depending of which is processed first (kinematic parameters for bottom-up approaches; goal for top-down approaches), selective grip and goal priming should be observed at short prime durations, with faster judgments for action pairs sharing either the same grip or the same goal. At long prime durations, both grip and goal representations should be activated and elicit action priming effects. In addition, effects of partial grip violations (e.g., grip incorrect but goal correct, see below stimuli section) or partial goal violations (e.g., grip correct but goal incorrect, see below stimuli section) should be visible: the co-activation of incongruent grip and goal information at long prime durations should interfere with target action processing. 


\section{EXPERIMENT 1}

\section{Materials and methods}

\section{Participants}

Thirty-one healthy participants took part in the study ${ }^{1}$. Three of them were left-handed or ambidextrous according to the Edinburgh handedness inventory (EHI; Oldfield, 1971) and were excluded. The final sample included 28 participants (mean age 22, age range 18-36, 23 females). All were right-handed (mean EHI= 87\%, from $45 \%$ to $100 \%$ ), reported normal or corrected-to-normal vision and provided written inform consent. They were not paid but could receive extra course credit for their participation. The protocol was approved by the Ethical Committee of the University and was in accordance with the declaration of Helsinki (1964, revised in 2013).

\section{Stimuli}

Twenty manipulable objects were selected (Appendix 1). Four $1024 * 683$ pixel coloured photographs of each reference object were taken, all involving the same right-handed actress interacting with the object in one of four conditions. The object-directed action could be correct or not by crossing the correctness of the grip used by the actress and the correctness of the action goal (Figure 1). Thus, there were four types of action stimuli: actions that were fully correct with no violation and actions that were incorrect owing to goal violations, grip violations, or both grip and goal violations. Correct grips were defined as the typical grasp-to-use grip of an object. Thus, an incorrect grip corresponded to an atypical (although not impossible) grip considering the typical use of the object. Similarly, correct goals were defined as the actor's goal to use the object in order to fulfil its typical/primary function. Thus, a goal was considered incorrect when the object could not be used for its typical function. Importantly, the incorrect grip did not prevent the correct goal to be achieved. Thus, grip and goal were manipulated independently. Note that contrary to van Elk et al. (2008) who manipulated goal representation in terms of goal location (e.g. bring cup to the mouth versus to the ear), we manipulate the orientation of the object to make its functional use possible or not, and then focus on the functional goal of the action (e.g., bring upright cup versus up-side down cup to the mouth). Moreover, the recipient object on which the object involved in the action may act upon (e.g. plant for water can) was not included in the picture to minimize expectancies that may be driven from other sources of information than the use of the tool.

\section{Experimental design}

Photographs were implemented in a priming paradigm. For each reference object, action photographs without violations ("correct" actions) and action photographs with both grip and goal violations (all "incorrect" actions) were used as targets for the "yes" (correct) and "no" (incorrect) judgment task. For each target, all four types of photographs were used as primes. Thus, each reference object was associated with 8 pairs of photographs divided in 4 types of action pairs. The prime could display the same grip and same goal (Grip and goal similar), a different grip but the same goal (Goal similar only), the same grip but a different goal (Grip similar only) or a different grip and a different goal (All different) as the target.

\footnotetext{
${ }^{1}$ A sample size of about 30 participants was chosen to ensure sufficient statistical power $(.80)$ for anticipated moderate effect sizes (Cohen $\mathrm{d}=.50$ for the critical paired comparisons).
} 
There was a total of 2 prime-target grip similarity (grip similar, grip different) x 2 prime-target goal similarity (goal similar, goal different) $\mathrm{x} 2$ response types (yes=correct target action, no=incorrect target action) $\mathrm{x}$ 20 objects $=160$ prime-target pairs. The design of the different priming conditions is presented on Figure 1.

Prime duration was manipulated in 2 conditions, a short $(66 \mathrm{~ms})$ and a long $(300 \mathrm{~ms})$ prime duration. The 160 pairs of photographs were then presented twice, once with a prime duration of $66 \mathrm{~ms}$ and once with a prime duration of $300 \mathrm{~ms}$, leading to 320 experimental trials. Trials were divided into 4 blocks of 80 trials, each block containing an equal number of trials of the 4 prime-target pairs $\mathrm{x} 2$ response types $\mathrm{x} 2$ prime duration conditions.

\section{Control measure of perceptual similarity}

An objective index of perceptual similarity between prime and target was computed using the FSIM algorithm (Zhang et al. 2011) to assess potential differences in terms of low-level visual features between primetarget pairs in the different conditions. According to this measure, pairs in the 'grip similar only' condition presented a higher degree of perceptual similarity than pairs in the 'goal similar only' and all different conditions $(\mathrm{p}=.001)$, the two having the same degree of perceptual similarity $(\mathrm{p}=.09)$, see also Figure 1 and Appendix 2 for details. Note that the perceptual similarity index in the grip and goal similar condition had no variance as the pair was composed of the same exact picture. Perceptual similarity scores were taken into account in a complementary analysis (below).

\section{[INSERT FIG 1]}

\section{Procedure}

Participants were seated at $100 \mathrm{~cm}$ from the screen so that the action in the picture would appear within 5 degrees of visual angle. Their task was to judge whether the action presented in the picture was correct or not (forced choice). Instructions were displayed on the screen and carefully explained by the experimenter to ensure that participants understood the correct/incorrect distinction. ${ }^{2}$ Twelve representative practice trials with feedback were provided with 3 objects that were not included in the actual experiment. The experimental session was the same as the practice session but without feedback. The experiment was conducted with E-Prime V2.0.10.353 software (Psychology Software Tools, Pittsburgh, PA).

Each trial began with a $2500 \mathrm{~ms}$ fixation cross, then a prime was displayed during 66 or $300 \mathrm{~ms}$, followed by a $66 \mathrm{~ms}$ grey screen mask, and finally the target until participants' response. Participants answered "yes" (correct) or "no" (incorrect) using their left and right hands on left and right extreme keys of the response box. Response mapping (yes/no, left/right) was counterbalanced between participants. Reaction times (RT) and correct responses (accuracy) were recorded. The four blocks and the 80 experimental trials within each block were randomly presented. Short breaks were proposed between blocks. The experiment lasted about 1 hour.

\section{Data Analysis}

\footnotetext{
2 “'On each trial, you will see two successive pictures showing an actor using an object. The first picture will always be briefly presented. You will have to judge the second photography. You will have to determine, as fast and as accurately as possible, if the presented action is correct or not according to the typical use of the object. The use of an object is atypical when the object is used for another purpose or in another manner as the typical one. You will start with a training in which you will have feedback."
} 
Correct RT were analysed as a function of grip similarity between prime and target (grip similar, grip different), goal similarity between prime and target (goal similar, goal different), prime duration (66ms, 300ms) and response type (yes, no); the three factors being repeated between participants and items. Response type was not a factor of interest but was introduced in the model since effect of yes/no response have been frequently reported. Effects of grip and goal similarities were expected to be modulated by prime duration. According to bottom-up approaches, grip information should be processed first and effect of grip similarity should be more and perhaps exclusively visible at $66 \mathrm{~ms}$, as compared to effect of goal similarity. Alternatively, according to top-down approaches, the goal should be activated first and effect of goal similarity should be more and perhaps exclusively obvious at $66 \mathrm{~ms}$, as compared to effect of grip similarity. For long latencies, observers should have activated both goal and grip information about the action regardless of whether observed actions are processed top-down or bottom up. Thus, both effects of grip and goal priming effects were expected at $300 \mathrm{~ms}$.

In brief, we expected an interaction between prime duration, grip priming and goal priming. The modulation of grip and goal priming by prime duration would reflect the different timing of activation of grip and goal information within the 66-300 ms time window. The interaction between grip and goal priming effects would reflect the detection of the incongruency between goal and grip information within the prime and would confirm that both grip and goal representations have been co-activated.

\section{Results}

\section{Data preparation for reaction times analysis}

All analyses were carried out with R version 3.3.2 (R Core Team 2017) and R Studio version 1.0.136. Overall, participants made few errors (mean $=2 \%$, range $.3 \%-6 \%$ ). Since the task was relatively easy to perform and errors were not of primary interest, errors were solely analysed in order to verify the absence of any trade-off effect. A chi-square test for independence indicated that errors were equally distributed between conditions $(\chi 2(3)$ $=.014, \mathrm{p}=.99$ ). Errors were then replaced by median RT of the remaining trials in each condition. RT superior to $1500 \mathrm{~ms}$ and inferior to $200 \mathrm{~ms}$ were considered outliers and then replaced by median RT of the remaining trials in each condition. Overall, $2.84 \%$ of the data were replaced. Final data are presented on Figure 2.

\section{[INSERT FIGURE 2]}

\section{Mixed-effect model analysis of correct action recognition RT}

A mixed-effect model comparison approach was used to analyse correct RT. This approach allows taking into account both items and participants as sources of variation and considering their possible interaction with the factors of interest (e.g., a given participant or a given item, may be more sensitive to grip similarity than another). Analyses were carried out with the lmer function of the lme4 1.1-12 package (Bates et al. 2015b).

The full model included grip similarity (GRIP), goal similarity (GOAL), prime duration (DURATION), response type (RESP) and related first-order, second-order, and third order interactions as fixed effects. It also included random intercepts and random slopes for GRIP, GOAL and RESP for participants and random intercepts for DURATION and RESP for items (1). The choice of this random effect structure resulted from a compromise 
between the maximum random effect structure (Barr et al. 2013) and the optimal random structure supported by the data (Bates, Kliegl, Vasishth, \& Baayen, 2015; Matuschek, Kliegl, Vasishth, Baayen, \& Bates, 2017; see Appendix $3 \mathrm{~A}$ for determination of the full model structure).

Augmented and reduced models (i.e., with and without the fixed effects of interest) were compared using 2LogLikelihood Ratio test distributed like $\chi^{2}$ with degrees of freedom corresponding to the number or parameters added. Parameter estimates of the models are provided with their corresponding t-values, when relevant. Pseudo $\mathrm{R}^{2}$ effect-sizes were computed with the r.squaredLR function of the MuMIn 1.15.6 package (Barton 2016). Note however that pseudo $\mathrm{R}^{2}$ for mixed-models cannot be interpreted using classical standards for effect size.

\section{Effect of grip and goal similarity priming}

The model comparison did not show any significant 4-way interaction between GRIP, GOAL, DURATION, and $\operatorname{RESP}\left(\chi^{2}(1)=.8861, \mathrm{p}=.346, \mathrm{R}^{2}=.10^{10^{\wedge-4}}\right)$. As expected, the 3-way interaction between GRIP, GOAL, and DURATION was significant $\left(\chi^{2}(1)=120.57, \mathrm{p}=.001, \mathrm{R}^{2}=133.67^{10^{\wedge-4}}\right)$. We thus tested the interaction model between GRIP and GOAL for each DURATION. At $66 \mathrm{~ms}$, there was no interaction between GRIP and GOAL $\left(\chi^{2}(1)=.11, p=.740, R^{2}=.25^{10^{\wedge}-4}\right)$. There was a main effect of GOAL $\left(\chi^{2}(1)=8.06, p=.004, R^{2}=17.98^{10^{\wedge}-4}\right.$; Estimate goal similar-goal different $=-8.53 \mathrm{~ms}, \mathrm{SE}=3 \mathrm{~ms}, \mathrm{t}=-2.84)$, but no significant main effect of GRIP $\left(\chi^{2}(1)=1.74, \mathrm{p}=.187, \mathrm{R}^{2}=3.89^{10^{\wedge}-4}\right.$; Estimate grip similar-grip different $\left.=-3.97 \mathrm{~ms}, \mathrm{SE}=3 \mathrm{~ms}, \mathrm{t}=-1.32\right)$ reflecting goal priming but no grip priming at $66 \mathrm{~ms}$. In contrast, at $300 \mathrm{~ms}$, the interaction model between GRIP and GOAL was significant $\left(\chi^{2}(1)=241.11, \mathrm{p}=.001, \mathrm{R}^{2}=523.96^{10^{\wedge-4}}\right)$. Parameter estimates of the model showed a main effect of GOAL (Estimate goal similar-goal different $=-15.71 \mathrm{~ms}, \mathrm{SE}=3.53 \mathrm{~ms}, \mathrm{t}=-4.44$ ) and a main effect of GRIP (Estimate grip similar-grip different $=-31.51 \mathrm{~ms}, \mathrm{SE}=2.90 \mathrm{~ms}, \mathrm{t}=-10.86$ ), reflecting both goal and grip priming at $300 \mathrm{~ms}$. In addition, the interaction estimate indicated that action primes with partial grip or goal violations slowed down target judgements by $62 \mathrm{~ms}$ in comparison with all similar or all different primes (estimate $=-61.78, \mathrm{SE}=3.92, \mathrm{t}=-15.74)$.

\section{Complementary analysis: influence of perceptual similarity}

Spearman's rank correlations were computed between the goal and grip similarity priming effects obtained in each response condition and the corresponding perceptual similarity indices. There were no significant correlations (all p > .66, see Appendix 2). 
This experiment used a priming paradigm involving object-direct action photographs with grip and/or goal violations to assess the relative timing of grip and goal activation during the processing of others' actions. The action goal was defined in functional terms and corresponded to the use of the object according to its typical function (e.g. water bottle: to drink). Action primes were expected to influence response times to determine whether target actions were correct or not when a) the prime shared grip or goal information with the target and when b) prime duration was sufficient to activate the common representation. We found that action primes with similar grip and similar goal both facilitated target action processing when presented for $300 \mathrm{~ms}$. Conversely, when action primes where presented for $66 \mathrm{~ms}$, only those sharing the same goal facilitated target action processing. Moreover, primes containing incongruent grip and goal information (partial violations) were more slowly processed that primes with no violation when presented for $300 \mathrm{~ms}$.

Priming effects for $300 \mathrm{~ms}$ prime duration are in line with previous experiments (van Elk et al. 2008; Kalénine et al. 2013) with the co-activation of both grip and goal representations during action decoding. Here, as long as participants had sufficient time to process the prime (300 ms), both grip and goal information influenced target action processing. Interestingly, the magnitude of grip similarity priming was greater than that of goal similarity priming. In our study, participants did not have to explicitly processed the different action dimensions manipulated in the primes. This suggests that whereas grip information may be more difficult to process when judged explicitly (cf. Van Elk et al., 2008), it strongly impacts action judgements when implicitly processed. Moreover, our priming paradigm highlights a cost on target processing when primes contained incongruent grip and goal information (partial action violations). This result mirrors the slower action decisions observed on action stimuli containing partial violations in previous studies (Kalénine et al., 2013; van Elk et al., 2008). In contrast to previous studies, participants were not explicitly instructed about the action dimensions manipulated. Thus, the cost entailed by partial action violations indicate that once activated, grip and goal representations automatically interact with one another during action perceptual processing. This extends the claim of the involvement of distinct but interrelated kinematic parameters and goal representations in perception.

When primes were presented for $66 \mathrm{~ms}$, only goal similarity priming was able to facilitate target action judgments. Absence of early grip priming may be interpreted in the light of previous studies that found that sensorimotor simulation of specific kinematic parameters performed by others may not arise before $200 \mathrm{~ms}$ (Naish, Houston-Price, Bremner, \& Holmes, 2014). Although modulations of corticospinal excitability by observed actions have been found as early as 90ms (e.g., Lepage et al. 2010; Cavallo et al. 2014 see Naish et al., 2014 for review), they would first correspond to a general preactivation of the motor system before reflecting the mapping of the specific kinematic parameters perceived onto the observer's own motor system. Therefore, even though the grip may have been processed during $66 \mathrm{~ms}$ of prime processing, this processing may have not been sufficient to influence target action judgments.

In contrast, early activation of goal-related information has been previously observed. In particular, EEG studies showed that discriminating between the different possible action goals of an observed action starts influencing brain activity after $60 \mathrm{~ms}$ of visual processing (e.g., Ortigue, Thompson, Parasuraman, \& Grafton, 2009). Yet these early responses have often been associated to perceptual processes (Catmur, 2015) rather than early activation of goal-related information. Earlier and stronger facilitative priming effects may then be expected between pairs of action pictures that are more perceptually similar. However, this possibility was ruled out using 
the FSIM measure of low-level perceptual similarity. Grip similar pairs were more perceptually similar than goal similar pairs and there was no correlation between the amplitude of grip and goal similarity priming effects and perceptual similarity differences between prime-target pairs. Thus, it is unlikely that the early facilitative goal priming effect merely reflects the greater perceptual similarity between action pairs in this condition. In contrast, goal priming results are consistent with an early activation of goal representations. Critically, goal activation was not only visible early but first. Thus, the relative timing of goal and grip activation supports a top-down processing of observed actions in our task. This suggests that participants first activate a prediction about the possible action goal of the actor that will guide the subsequent processing of the kinematic parameters of the action, which occurred between 60 and $300 \mathrm{~ms}$.

To further specify the time-course of grip and goal activation, and in particular determine the minimal time required to activate both grip and goal representations from action visual stimuli, a second Experiment was carried out. Intermediate prime durations were selected between 66 and $300 \mathrm{~ms}$. A first duration of $120 \mathrm{~ms}$ was chosen, which corresponds to the late boundary of first time window of action understanding identified at the neurophysiological level (e.g., Ortigue et al., 2009). At 120 ms, facilitative effects of goal similarity were expected, which would confirm the earliness of goal activation. A second prime duration of $220 \mathrm{~ms}$ was selected to fit the timing at which specific motor responses have been reported during action observation (Naish et al., 2014). Effects of grip similarity should emerge between 120 and $220 \mathrm{~ms}$. Experiment 2 followed the same rationale and paradigm as Experiment, asides from two additions:

a) The strength of the perceptual mask used between prime and target was enhanced for a greater control of lowlevel perceptual priming effects.

b) A neutral prime condition presenting the object without action was added to further evaluate the role of object information in priming effects involving object-related actions. 


\section{EXPERIMENT 2}

\section{Materials and methods}

\section{Participants}

Thirty healthy participants took part in the study. Five of them were classified as left-handed or ambidextrous according to the EHI (Oldfield 1971), and were excluded from the study. The final sample included 25 participants (mean age 21, age range 18-30, 16 females). All were right-handed (mean EHI= 84\%, from $43 \%$ to $100 \%$ ), reported normal or corrected-to-normal vision and provided written inform consent. They were not paid but could receive extra course credit for their participation. The protocol was approved by the Ethical Committee of the University and was in accordance with the declaration of Helsinki (1964, revised in 2013).

\section{Stimuli}

Experiment 2 used the same prime-target action pairs as Experiment 1, divided in 4 conditions: 'Grip and goal similar', 'Goal similar only', 'Grip similar only', and 'All different'. Furthermore, a control condition was added in which the target was coupled with a neutral action-free prime in which the object was displayed in front of the actress with her hands resting on the table (Neutral). This control condition was designed to evaluate to what extent mere object priming could account for the expected effects of goal and/or grip similarity priming (see complementary analysis below). Overall, there was a total of 5 prime-target pairs x 2 response types x 20 objects $=200$ prime-target pairs (see Figure 1, and Appendix 1). Trials were divided into 4 blocks of 100 trials, each bloc containing an equal number of trials of the 5 prime-target pairs $\mathrm{x} 2$ response types $\mathrm{x} 2$ prime duration conditions.

\section{Procedure}

Experiment 2 evaluated two intermediate prime durations of $120 \mathrm{~ms}$ and $220 \mathrm{~ms}$. A few additional changes were applied to the procedure. The grey mask used in the first experiment was replaced by a black and white pixelated mask and the target picture was spatially shifted from the prime picture by 0.5 degree of visual angle. These subtle changes have been made to the procedure in order to provide a stronger control of low-level visual effects such as visual persistence and low-level spatial overlap. Apart from those modifications, the procedure and the rationale of Experiment 2 were the same as Experiment 1. The four blocks and the 100 experimental trials within each block were randomly presented. Short breaks were proposed between blocks. The experiment lasted about 1 hour.

\section{Data analysis}

As in Experiment 1, RTs were analysed as a function of grip similarity between prime and target (grip similar, grip different), goal similarity between prime and target (goal similar, goal different), prime duration $(120 \mathrm{~ms}, 220 \mathrm{~ms})$ and response type (yes, no); the three factors being repeated between participants and items. As goal similarity priming was observed from $66 \mathrm{~ms}$ of prime duration in Experiment 1, it was expected at both 120 ms and $220 \mathrm{~ms}$ of prime duration in Experiment 2. In contrast, as grip similarity priming was evidenced only for $300 \mathrm{~ms}$ prime duration in Experiment 1, grip decoding was supposed to occur sometime between 66 and $300 \mathrm{~ms}$ and grip priming effects could possibly not be visible at one or both of these intermediate prime durations. 
As in Experiment 1, we expected an interaction between prime duration, grip priming and goal priming. The modulation of grip and goal priming by prime duration would reflect the different timing of activation of grip and goal information within the 120-220 ms time window. Again, the interaction between grip and goal priming effects would reflect the detection of the incongruency between goal and grip information within the prime and would confirm that both grip and goal representations have been co-activated.

\section{Results}

\section{Data preparation for reaction times analysis}

Again, error rate was relatively low (mean $=3 \%$, from $0.4 \%$ to $12 \%$ ). A chi-square test for independence revealed that errors were equally distributed between conditions $(\chi 2(4)=.0126, p>.99)$. Errors were replaced by median RT of the remaining items in each condition. RT superior to $1500 \mathrm{~ms}$ and inferior to $200 \mathrm{~ms}$ were considered outliers and then replaced by median RT of the remaining items in each condition. Overall, $3.1 \%$ of the data were replaced. Final data are reported on Figure 2.

\section{Mixed-effect model analysis of correct action recognition RT}

The same mixed-effect model approach was used to analyse correct RT in Experiment 2. The full model included grip similarity (GRIP), goal similarity (GOAL), prime duration (SOA) and response type (RESP) and related first-order, second-order and third order interactions as fixed effects. It also included random intercepts and random slopes for RESP for participants and intercept and random slopes for DURATION and RESP for items (2). The final model structure is justified in Appendix 3B.

\section{Effect of grip and goal similarity priming}

Comparison of augmented and reduced models highlighted a 4-way interaction between GRIP, GOAL, DURATION and $\operatorname{RESP}\left(\chi^{2}(1)=3.82, \mathrm{p}=.051, \mathrm{R}^{2}=4.78^{10^{\wedge}-4}\right)$. We therefore tested the interaction between GRIP, GOAL, and DURATION for each response type separately.

For YES responses, the GRIP $x$ GOAL $x$ DURATION interaction was not significant $\left(\chi^{2}(1)=1.34, p=.246\right.$, $\left.\mathrm{R}^{2}=3.36^{10^{\wedge-4}}\right)$. The GRIP $\times$ GOAL interaction was thus evaluated regardless of prime duration for yes responses. The GRIP $x$ GOAL interaction model was significant $\left(\chi^{2}(1)=34.04, \mathrm{p}=.001, \mathrm{R}^{2}=84.74^{10^{\wedge}-4}\right)$. Parameter estimates of this model showed a main effect of GOAL (Estimate goal similar-goal different $=-33.83 \mathrm{~ms}, \mathrm{SE}=2.96 \mathrm{~ms}, \mathrm{t}$ $=-11.43$ ) and a main effect of GRIP (Estimate grip similar-grip different $=-20.44 \mathrm{~ms}, \mathrm{SE}=2.96 \mathrm{~ms}, \mathrm{t}=-6.91$ ), reflecting both goal and grip priming at intermediate prime durations. In addition, the interaction estimate indicated that action primes with partial grip or goal violations slowed down target judgements by $32 \mathrm{~ms}$ in comparison with primes that were all similar or all different (estimate $=-32.00, \mathrm{SE}=4.07, \mathrm{t}=-7.87$ ).

For NO responses, the GRIP x GOAL $x$ DURATION interaction was significant $\left(\chi^{2}(1)=16.21, p=.001, \mathrm{R}^{2}\right.$ $\left.=39.97^{10^{\wedge}-4}\right)$. The GRIP $x$ GOAL interaction model was significant at $120 \mathrm{~ms}\left(\chi^{2}(1)=6.86, \mathrm{p}=.008, \mathrm{R}^{2}=34.24\right.$ 10^-4). Parameter estimates of this model did not show any main effect of GOAL or GRIP (Estimate goal similargoal different $=-0.38 \mathrm{~ms}, \mathrm{SE}=4.25 \mathrm{~ms}, \mathrm{t}=-0.09$; Estimate grip similar-grip different $=-3.78 \mathrm{~ms}, \mathrm{SE}=4.25 \mathrm{~ms}$, $\mathrm{t}=-0.89)$. Yet the interaction estimate indicated that action primes with partial grip or goal violations slowed down 
target judgements by $16 \mathrm{~ms}$ in comparison with primes that were all similar or all different (estimate $=-15.75, \mathrm{SE}=$ $6.01, \mathrm{t}=-2.62)$. The GRIP x GOAL interaction model was also significant at $220 \mathrm{~ms}\left(\chi^{2}(1)=76.66, \mathrm{p}=.001, \mathrm{R}^{2}\right.$ $=376.04^{10^{\wedge}-4}$ ). Parameter estimates of the model demonstrated a main effect of GOAL (Estimate goal similar-goal different $=-13.41 \mathrm{~ms}, \mathrm{SE}=3.86 \mathrm{~ms}, \mathrm{t}=-3.47)$ and a main effect of grip (Estimate grip similar-grip different $=-$ $17.79 \mathrm{~ms}, \mathrm{SE}=3.86 \mathrm{~ms}, \mathrm{t}=-4.61$ ). The interaction estimate showed a cost of $48 \mathrm{~ms}$ on target judgements for action primes with partial grip or goal violations compared to primes that were all similar or all different (estimate $=-48.26, \mathrm{SE}=5.46, \mathrm{t}=-8.84)$.

Together, results of Experiment 2 showed effects of goal and grip similarity from $120 \mathrm{~ms}$ of prime processing. Interactions with response type and prime duration suggest a subtle additional modulation of grip and goal similarity effects with prime duration $(120,220 \mathrm{~ms})$ in the case of no-responses. We will go back to these nuances in the Discussion of Experiment 2.

\section{[INSERT TABLE 1]}

\section{Complementary analysis: Effect of object priming}

If object identity is used to derive information about the goal or grip of the action, then the pattern of goal and grip similarity priming effects should follow the pattern of object priming effects. To evaluate this hypothesis, we replaced the conditions in which grip or goal overlapped between prime and target with the neutral priming condition. In other words, neutral prime-target pairs (object similar) were compared with prime-target pairs that differed on grip (grip dissimilar conditions) on the hand, and with prime-target pairs that differed on goal (goal dissimilar conditions, see Table 1). This comparison was tested in situations where we observed an effect of grip similarity and goal similarity in the main analysis of Experiment 2.

For YES responses, object similarity did not show any advantage over grip dissimilarity $\left(\chi^{2}(1)=1.02, p\right.$ $=.312, \mathrm{R}^{2}=2.55^{10^{\wedge}-4}$ ). This stands in contrast with the grip similarity priming observed in the main analysis and indicates that object repetition is not responsible of the grip similarity priming effect. Object similarity did show a $14 \mathrm{~ms}$ advantage over goal dissimilarity, however $\left(\chi^{2}(1)=10.43, \mathrm{p}=.002, \mathrm{R}^{2}=26.03^{10^{\wedge}-4,}\right.$; estimate $=-13.83, \mathrm{SE}$ $=4.28, \mathrm{t}=-3.23)$, suggesting that object similarity priming could at least partially account for the effect of goal similarity in the main analysis.

For NO responses, object similarity was systematically less facilitative than grip similarity (at $120 \mathrm{~ms}$ : $\chi^{2}(1)=12.06, \mathrm{p}=.001, \mathrm{R}^{2}=60.13^{10^{\wedge}-4,}$; estimate $=+13.32, \mathrm{SE}=3.83, \mathrm{t}=3.48 ;$ at $220 \mathrm{~ms}: \chi^{2}(1)=23.95, \mathrm{p}=.001$, $\mathrm{R}^{2}=119.05^{10^{\wedge}-4,}$; estimate $\left.=+19.32, \mathrm{SE}=3.93, \mathrm{t}=4.91\right)$ and goal similarity $\left(\right.$ at $120 \mathrm{~ms}: \chi^{2}(1)=19.94, \mathrm{p}=.001$, $\mathrm{R}^{2}=84.32^{10^{\wedge}-4, ;}$ estimate $=+21.24, \mathrm{SE}=5.15, \mathrm{t}=4.12 ;$ at $220 \mathrm{~ms}: \chi^{2}(1)=28.73, \mathrm{p}=.001, \mathrm{R}^{2}=143.00^{10^{\wedge}-4, ;}$ estimate $=+30.42, \mathrm{SE}=5.65, \mathrm{t}=5.38$ ). Facilitative effects of grip or goal similarity for no responses were thus not due to object similarity priming.

\section{Complementary analysis: influence of perceptual similarity}

As in the Experiment 1, Spearman's rank correlations were computed between the goal and grip similarity priming effects obtained in each response condition and the corresponding perceptual similarity indices. There were no significant correlations (all p > .29, see Appendix 2). 


\section{Discussion}

The second experiment demonstrates the activation of both grip and goal representations from $120 \mathrm{~ms}$ of processing of the action photographs. We used the same priming paradigm as in Experiment 1 with different prime durations (120 and $220 \mathrm{~ms}$ ) and with the addition of a neutral control prime condition (actor and object present without any performed action) to assess the role of object information in the action priming effects observed. Overall, we found that the recognition of correct actions was facilitated when action primes shared the same action goal or the same grip as the target. This facilitation was visible from $120 \mathrm{~ms}$ of prime processing for "yes" responses and the magnitude of the action priming effect was more important for goal than for grip similarity. For "no" responses, goal and grip similarity priming effects were only significant at $220 \mathrm{~ms}$.

The effect of response type in Experiment 2, although not predicted, is not at odds with the literature on action processing (e.g., Yoon, Humphreys, \& Riddoch, 2010). Van Elk et al. (2008) also found an effect of response type, with an impact of action violations on action decisions only when the dimension of the action evaluated (correctness of grip or correctness of goal) was correct. This may suggest that regardless of the action dimension considered, correct representations have a greater weight than incorrect representations in the comprehension of observed actions. In our experiment, goal and grip similarity priming effects were not completely absent for "no" responses, but the emergence of both goal and grip priming effects was slightly delayed and becomes visible from 220 ms of prime duration, in contrast with priming effects for "yes" responses evidenced regardless of prime duration from $120 \mathrm{~ms}$. Although a better understanding of the reasons underlying this delay is needed, the effect of response type on action priming effects does not impact the main result of Experiment 2, namely the appearance of grip similarity priming from $120 \mathrm{~ms}$ of prime processing, and the consistency of the whole data pattern between Experiments 1 and 2. At extremely short and long prime durations (Experiment 1), the effect of response type was not significant since at $66 \mathrm{~ms}$ prime duration was far too short to trigger grip similarity priming and at $300 \mathrm{~ms}$ grip similarity priming was already well established. Response type affected the prime durations at which grip similarity priming became obvious, between 120 and $220 \mathrm{~ms}$ (Experiment 2).

The main finding of Experiment 2, particularly clearly highlighted for "yes" responses, shows both grip and goal similarity priming effects from $120 \mathrm{~ms}$ of prime processing, with a greater magnitude of goal priming effects. Note that moreover, as in Experiment 1, a cost for primes conveying incongruent grip and goal information (i.e., primes with partial violations) was observed from $120 \mathrm{~ms}$ regardless of response type, as reflected by significant interactions between grip and goal similarity. Since both grip and goal representations have to be activated in order to induce a cost when incongruent, this effect reinforces the idea that grip similarity priming emerges as early as $120 \mathrm{~ms}$. Again, we can be fairly confident that differences in low-level perceptual similarity between prime-target pairs cannot account for the pattern of priming effects. The stronger masking procedure used in Experiment 2 minimized the possible influence of low-level similarity priming and, more importantly, there was no correlation between the amplitude of grip and goal priming effects and perceptual similarity indices in the different conditions (Appendix 2).

Finally, the complementary analysis involving the neutral prime condition indicated that prime-target pairs sharing mere object similarity showed an advantage over prime-target pairs with dissimilar goals, but not dissimilar grips (in the "yes" response condition). This suggests that the advantage of prime-target pairs sharing goal similarity over prime-target pairs with dissimilar goals (i.e., the goal priming effect in the main analysis) could be due to mere object priming. However, the magnitude of the goal priming effect (34 ms) was twice as big as the object 
priming effect (14 ms), indicating that object priming may only partially account for the goal similarity priming effects observed for "yes" responses. One interpretation of this additional result is that observers may use some information about object identity to activate a prediction about the goal of the action. We will go back to this interpretation in the General Discussion.

\section{General discussion}

Whereas the involvement of grip and goal representations during the processing of others' actions is well accepted, their specific role and temporal organization remain debated. Bottom-up approaches propose that action decoding is first guided by the processing of kinematic parameters, whereas top-down approaches suggest that predictions about others' goal precede the use of kinematic information. The present study evaluates these hypotheses by assessing the timing of grip and goal activation during correctness judgments of photographs of object-directed actions. The selective activation of grip or goal representations was identified using action primes sharing the same grip or the same goal as target actions. Information about activation time course was captured by varying prime duration (66 ms and $300 \mathrm{~ms}$ in Experiment 1; $120 \mathrm{~ms}$ and $220 \mathrm{~ms}$ in Experiment 2). Goal similarity priming was visible for all prime durations whereas grip priming emerged from $120 \mathrm{~ms}$ of prime duration. Interestingly, action primes conveying incongruent grip and goal information interfered with action recognition when there was enough time for grip and goal representations to be co-activated, namely from $120 \mathrm{~ms}$. These results indicate that goal representations are activated first and provide additional evidence for distinct but complementary roles of grip and goal representations during the processing of others' actions.

Top-down approaches (Kilner 2011) have been supported by recent studies highlighting the involvement of contextual information in action understanding (Iacoboni et al. 2005; Giglio et al. 2013; Amoruso and Urgesi 2016; Wurm and Schubotz 2016; Schenke et al. 2016). In such studies, contextual information is provided before the presentation of the kinematic parameters of an action. They show that congruent context helps determining action goals from observed kinematics. This demonstrates that contextual information can shape a priori predictions of the observer about the goal of the action, which will influence the processing of kinematic parameters. However, these studies do not allow determining at what moment of action processing a representation of the action goal is activated in the absence of prior contextual information providing externally-driven expectancies. In our protocol, information about the goal and the kinematics of object-directed actions is presented simultaneously in action photographs. The hand grip on the object provides information about the kinematics of the action (e.g. grasp water bottle) and the orientation of the object informs about the goal of the action (e.g. drink). Thus, timing differences between goal and grip priming effects reflect differences in the time course of activation of representations of action goals (e.g., representation of the object typical function) and kinematic parameters (i.e. representation of the functional hand posture for object use) from the perceived object-related action. Our study then makes a unique contribution by demonstrating the early and first activation of goal representations.

The photographs of object use actions involved the hand effector on the tool. One may argue that the object is a context per se from which goal expectancies could be driven. Goals expectancies driven from the object may thus lead to faster activation of goal representations. Although we consider the tool object an integral part of object-directed actions, we acknowledge that prediction about the action goal can be driven from object identity (e.g. water bottle: to drink). Yet this is also true for predictions regarding the grip parameter (e.g. water bottle: 
whole hand clench). Consistent with the idea, recent theoretical assumptions assign a central role to object information in both execution and perception of object-directed actions (Bach et al. 2014; van Elk et al. 2014). In Experiment 2, mere object similarity priming showed a similar advantage as goal similarity priming, indicating that processing of object identity may have led to the activation of a set of potential goals corresponding to object typical use. Importantly, however, processing of object identity cannot fully account for the differences between goal and grip priming effects. First, the magnitude of goal similarity priming was twice as big as the magnitude of object priming. Second, grip similarity priming, in contrast, was not similar to object priming even if the object could easily activate grip representations. Together, the pattern of results demonstrate that object-directed actions tend to be processed top-down in accordance to predictive accounts of action understanding. The observer uses the available information to make first a prediction about the goal of the action (rather than a prediction about the grip of the action) that will be then confronted to the processing of the kinematic parameters.

Finally, the late activation of grip representations in our experiments adds to the inconsistent and widely discussed literature on the role of kinematic information in the processing of observed actions. Some evidence clearly indicates that processing of kinematic parameters fuels decisions about action goals (Ansuini et al. 2014; Quesque and Coello 2015; Cavallo et al. 2016) . Still, despite the poor contextual information provided, kinematic processing may still be guided by predictions about possible action goals, especially in studies requiring decisions between a limited subset of possible goals. In Lewkowicz et al. (2015) for example, video clips displayed an actor's arm reaching and moving a dowel in order to move it away or take it for himself. Observers may first pre-activate the only two possible goals (move away versus take it) and then use kinematics information to verify each hypothesis. Actions would be processed in a predictive manner. Finally, the use of kinematic information in action understanding may be flexible, context- and task-dependent. Some experiments have found that on-line kinematic information is not necessary or not always used in the processing of observed actions (Longo et al. 2008; Neal and Kilner 2010; Naish et al. 2013; Geangu et al. 2015). In parallel, reliance on kinematic information is increased when the object is absent (Thioux and Keysers 2015), when the action is difficult to recognize (Nicholson et al. 2017) or when the recognition of the action requires the processing of fine-grained kinematic information (Tidoni et al. 2013). Thus, a strong possibility is that actions performed by others can be flexibly decoded through topdown or bottom-up mechanisms, depending of contextual variables (e.g., object presence, visual conditions, contextual scene, social factors, or even kinematic information provided by others' actions see for example Manera et al. 2011) and task demands.

Limitations to the study

There are a few limitations to this study that deserve consideration. First, participant samples were not gender balanced and although there was no obvious a priori or a posteriori evidence of gender effects, the possibility of gender modulation of action processing cannot be ruled out. Second, the set of object stimuli was relatively heterogeneous and included both tools (objects related to specific usage) and non-tools (objects with many possible usages). It is thus difficult to determine from the present study whether object status has an impact on action decoding. Third, we used static action stimuli for a strict control of grip and goal visual information input. This leaves the issue of the generalization of the present findings to naturalistic, dynamic action perception open. Last but not least, although grip and goal information were manipulated independently in the present design, some asymmetry in the relation between the two pieces of information remains: an incorrect grip can still be used 
1 to achieve a correct action, as defined as the typical use of the object (e.g., water bottle: grasp-to-drink), whereas

2 an incorrect goal (e.g., water bottle up-side down) prevents the achievement of this action. This is not a strict

3 asymmetry, however, since other possible and meaningful actions may be achieved in the incorrect goal conditions,

4 even if less probable and/or less associated to object function (e.g., grasp-to-move actions when transporting

5 objects are possible regardless of object orientation). In order to evaluate whether goal information remains first

6 processed when it is less predictive of action correctness, future research could attempt to cross two alternatives

7 (and ideally equally likely) grips and goals for a given object (e.g. pour versus drink from cup).

\section{Conclusion}

To conclude, the present study provides evidence for the activation of distinct goal and kinematic parameters representations within the first $120 \mathrm{~ms}$ of processing of observed actions, with an early and first activation of goal representations. Results also suggest that early goal representations may have been at least partially derived from information related to object identity. These results are in line with top-down predictive approaches of action understanding in which a prediction about the action goal is first computed and then compared with the processing of kinematic parameters. Future research should examine to what extent the timing of goal and grip activation is sensitive to context and task demands. Context-dependent changes in grip and goal temporal dynamics would support the hypothesis of flexible bottom-up and top-down mechanisms in action decoding. 


\section{Acknowledgments}

This work was funded by the French National Research Agency (ANR-16-CE28-0003 and ANR-11EQPX-0023) and benefited from a regional fellowship (Hauts-de-France) to J. Decroix.

\section{References}

Amoruso L, Urgesi C (2016) Familiarity modulates motor activation while other species' actions are observed: A magnetic stimulation study. Eur J Neurosci 43:765-772. doi: 10.1111/ejn.13154

Ansuini C, Cavallo A, Bertone C, Becchio C (2014) The visible face of intention: Why kinematics matters. Front Psychol 5:1-6. doi: 10.3389/fpsyg.2014.00815

Avanzini P, Fabbri-Destro M, Campi C, et al (2013) Spatiotemporal dynamics in understanding hand-object interactions. Proc Natl Acad Sci U S A 110:15878-85. doi: 10.1073/pnas.1314420110

Bach P, Nicholson T, Hudson M (2014) The affordance-matching hypothesis: how objects guide action understanding and prediction. Front Hum Neurosci 8:254. doi: 10.3389/fnhum.2014.00254

Barr DJ, Levy R, Scheepers C, Tily HJ (2013) Random effects structure for confirmatory hypothesis testing: Keep it maximal. J Mem Lang 68:255-278. doi: 10.1016/j.jml.2012.11.001

Barsalou LW (2008) Grounded Cognition. Annu Rev Psychol 59:617-645. doi: 10.1146/annurev.psych.59.103006.093639

Barton K (2016) MuMIn: Multi-Model Inference.

Bates D, Kliegl R, Vasishth S, Baayen H (2015a) Parsimonious mixed models. arXiv Prepr arXiv150604967 127. doi: arXiv:1506.04967

Bates D, Mächler M, Bolker B, Walker S (2015b) Fitting Linear Mixed-Effects Models Using Ime4. J Stat Softw. doi: 10.18637/jss.v067.i01

Catmur C (2015) Understanding intentions from actions: Direct perception, inference, and the roles of mirror and mentalizing systems. Conscious Cogn 1-8. doi: 10.1016/j.concog.2015.03.012

Cattaneo L, Sandrini M, Schwarzbach J (2010) State-dependent TMS reveals a hierarchical representation of observed acts in the temporal, parietal, and premotor cortices. Cereb Cortex 20:2252-2258. doi: 10.1093/cercor/bhp291

Cavallo A, Heyes C, Becchio C, et al (2014) Timecourse of mirror and counter-mirror effects measured with transcranial magnetic stimulation. Soc Cogn Affect Neurosci 9:1082-1088. doi: 10.1093/scan/nst085

Cavallo A, Koul A, Ansuini C, et al (2016) Decoding intentions from movement kinematics. Sci Rep 6:37036. doi: $10.1038 /$ srep37036

Geangu E, Senna I, Croci E, Turati C (2015) The effect of biomechanical properties of motion on infants' perception of goal-directed grasping actions. J Exp Child Psychol 129:55-67. doi: 10.1016/j.jecp.2014.08.005 
Gentsch A, Weber A, Synofzik M, et al (2016) Towards a common framework of grounded action cognition: Relating motor control, perception and cognition. Cognition 146:81-89.

Giglio ACA, Minati L, Boggio PS (2013) Throwing the banana away and keeping the peel: Neuroelectric responses to unexpected but physically feasible action endings. Brain Res 1532:56-62. doi: 10.1016/j.brainres.2013.08.017

Grafton ST, Hamilton AFDC (2007) Evidence for a distributed hierarchy of action representation in the brain. Hum Mov Sci 26:590-616. doi: 10.1016/j.humov.2007.05.009

Hudson M, Nicholson T, Ellis R, Bach P (2016a) I see what you say: Prior knowledge of other's goals automatically biases the perception of their actions. Cognition 146:245-250. doi: 10.1016/j.cognition.2015.09.021

Hudson M, Nicholson T, Simpson WA, et al (2016b) One step ahead: The perceived kinematics of others' actions are biased toward expected goals. J Exp Psychol Gen 145:1-7. doi: 10.1037/xge0000126

Iacoboni M, Molnar-Szakacs I, Gallese V, et al (2005) Grasping the intentions of others with one's own mirror neuron system. PLoS Biol 3:0529-0535. doi: 10.1371/journal.pbio.0030079

Jacquet PO, Avenanti A (2015) Perturbing the action observation network during perception and categorization of actions' goals and grips: State-dependency and virtual lesion TMS effects. Cereb Cortex 25:598-608. doi: 10.1093/cercor/bht242

Kalénine S, Shapiro AD, Buxbaum LJ (2013) Dissociations of action means and outcome processing in lefthemisphere stroke. Neuropsychologia 51:1224-1233. doi: 10.1016/j.neuropsychologia.2013.03.017

Kilner JM (2011) More than one pathway to action understanding. Trends Cogn Sci 15:352-357. doi: 10.1016/j.tics.2011.06.005

Kilner JM, Friston KJ, Frith CD (2007) Predictive coding: An account of the mirror neuron system. Cogn Process 8:159-166. doi: 10.1007/s10339-007-0170-2

Kristjansson A (2008) "I know what you did on the last trial" - a selective review of research on priming in visual search. Front Biosci 13:1171. doi: 10.2741/2753

Lepage JF, Tremblay S, Théoret H (2010) Early non-specific modulation of corticospinal excitability during action observation. Eur J Neurosci 31:931-937. doi: 10.1111/j.1460-9568.2010.07121.x

Longo MR, Kosobud A, Bertenthal BI (2008) Automatic imitation of biomechanically possible and impossible actions: effects of priming movements versus goals. J Exp Psychol Hum Percept Perform 34:489-501. doi: 10.1037/0096-1523.34.2.489

Manera V, Becchio C, Schouten B, et al (2011) Communicative interactions improve visual detection of biological motion. PLoS One. doi: 10.1371/journal.pone.0014594

Matuschek H, Kliegl R, Vasishth S, et al (2017) Balancing Type I error and power in linear mixed models. J Mem Lang 94:305-315. doi: 10.1016/j.jml.2017.01.001 
Naish KR, Houston-Price C, Bremner AJ, Holmes NP (2014) Effects of action observation on corticospinal excitability: Muscle specificity, direction, and timing of the mirror response. Neuropsychologia 64:331348. doi: 10.1016/j.neuropsychologia.2014.09.034

Naish KR, Reader AT, Houston-Price C, et al (2013) To eat or not to eat? Kinematics and muscle activity of reach-to-grasp movements are influenced by the action goal, but observers do not detect these differences. Exp Brain Res 225:261-275. doi: 10.1007/s00221-012-3367-2

Neal A, Kilner JM (2010) What is simulated in the action observation network when we observe actions? Eur J Neurosci 32:1765-1770. doi: 10.1111/j.1460-9568.2010.07435.x

Nicholson T, Roser M, Bach P (2017) Understanding the goals of everyday instrumental actions is primarily linked to object, not motor-kinematic, information: Evidence from fMRI. PLoS One 12:1-21. doi: 10.1371/journal.pone.0169700

Oldfield RC (1971) The assessment and analysis of handedness: The Edinburgh inventory. Neuropsychologia 9:97-113. doi: 10.1016/0028-3932(71)90067-4

Ortigue S, Thompson JC, Parasuraman R, Grafton ST (2009) Spatio-temporal dynamics of human intention understanding in temporo-parietal cortex: A combined EEG/fMRI repetition suppression paradigm. PLoS One. doi: 10.1371/journal.pone.0006962

Quesque F, Coello Y (2015) Perceiving what you intend to do from what you do: evidence for embodiment in social interactions. Socioaffective Neurosci Psychol 5:28602. doi: 10.3402/snp.v5.28602

R Core Team (2017) R: A language and environment for statistical computing. R Foundation for Statistical Computing, Vienna

Rizzolatti G, Fogassi L (2014) The mirror mechanism: recent findings and perspectives. Philos Trans R Soc Lond B Biol Sci 369:20130420. doi: 10.1098/rstb.2013.0420

Schenke KC, Wyer NA, Bach P (2016) The Things You Do: Internal Models of Others' Expected Behaviour Guide Action Observation. PLoS One 11:e0158910. doi: 10.1371/journal.pone.0158910

Thioux M, Keysers C (2015) Object visibility alters the relative contribution of ventral visual stream and mirror neuron system to goal anticipation during action observation. Neuroimage 105:380-394. doi: 10.1016/j.neuroimage.2014.10.035

Tidoni E, Borgomaneri S, di Pellegrino G, Avenanti A (2013) Action simulation plays a critical role in deceptive action recognition. J Neurosci 33:611-23. doi: 10.1523/JNEUROSCI.2228-11.2013

van Elk M, Bousardt R, Bekkering H, van Schie HT (2012) Using goal- and grip-related information for understanding the correctness of other's actions: An ERP study. PLoS One 7:1-8. doi: 10.1371/journal.pone.0036450

van Elk M, van Schie H, Bekkering H (2014) Action semantics: A unifying conceptual framework for the selective use of multimodal and modality-specific object knowledge. Phys Life Rev 11:220-250. doi: 
van Elk M, Van Schie HT, Bekkering H (2008) Conceptual knowledge for understanding other's actions is organized primarily around action goals. Exp Brain Res 189:99-107. doi: 10.1007/s00221-008-1408-7

Wolpert D, Doya K, Kawato M (2003) A unifying computational framework for motor control and social interaction. Philos Trans R Soc Lond B Biol Sci 358:593-602. doi: 10.1098/rstb.2002.1238

Wurm MF, Lingnau A (2015) Decoding Actions at Different Levels of Abstraction. J Neurosci 35:7727-7735. doi: 10.1523/JNEUROSCI.0188-15.

Wurm MF, Schubotz RI (2012) NeuroImage Squeezing lemons in the bathroom : Contextual information modulates action recognition. Neuroimage 59:1551-1559. doi: 10.1016/j.neuroimage.2011.08.038

Wurm MF, Schubotz RI (2016) What's she doing in the kitchen? Context helps when actions are hard to recognize. Psychon Bull Rev 1-7. doi: 10.3758/s13423-016-1108-4

Yoon EY, Humphreys GW, Riddoch MJ (2010) The paired-object affordance effect. J Exp Psychol Hum Percept Perform 36:812-824. doi: 10.1037/a0017175

Zentgraf K, Munzert J, Bischoff M, Newman-Norlund RD (2011) Simulation during observation of human actions - Theories, empirical studies, applications. Vision Res 51:827-835. doi: 
1 APPENDIX 1: List of objects used in Experiments 1 and 2

\begin{tabular}{|l|}
\hline Carafe \\
\hline Coffee cup \\
\hline Fork \\
\hline Hairbrush \\
\hline Hairdryer \\
\hline Hammer \\
\hline Knife \\
\hline Liquid soap \\
\hline Magnifying glass \\
\hline Pencil \\
\hline Phone \\
\hline Screwdriver \\
\hline Tea spoon \\
\hline Teapot \\
\hline Toothbrush \\
\hline Torch \\
\hline Cream Tube \\
\hline Water bottle \\
\hline Watering can \\
\hline Wine glass \\
\hline
\end{tabular}

2 
2 Priming paradigms are known to be influenced by low-level visual features (e.g., Kristjansson, 2008). Thus, our 3 results may be driven by perceptual differences between conditions and not by the activation of different levels of 4 action representation. For example, earlier and stronger priming effects may be expected between pairs of action 5 pictures that are more similar perceptually. We used the FSIM algorithm developed by Zhang, Zhang, Mou \& 6 Zhang (2011) to assess image similarity based on low-level visual features. The closer to 1 the index is the more 7 pictures are similar perceptually. First, an index of perceptual similarity was computed between each type of prime 8 type (correct grip but incorrect goal, incorrect grip but correct goal, incorrect grip and incorrect goal, neutral action9 free) and target (correct action target, incorrect action target) and then attributed to each type of prime-target pairs 10 (Grip similar only, goal similar only, all different, neutral). No indices were computed for grip and goal similar pairs, as prime and target were the same exact picture.

Perceptual similarity was modelled as a function of prime-target pairs (Grip similar only, goal similar only, all different, neutral) and RESP (yes, no) as fixed effects and items as random intercepts. Model comparison did not show any interaction between RESP and type of pair $\left(\chi^{2}(3)=2.5356, \mathrm{p}=0.4689\right)$ and no main effect of RESP $\left(\chi^{2}(1)=1.7477, p=0.1862\right)$. We did observe however a main effect of type of pair $\left(\chi^{2}(3)=213.11, p<0.001\right)$. Compared to 'all different' pairs, perceptual similarity was higher in 'similar grip only' pairs (estimate $=0.055$, $\mathrm{SE}=0.004, \mathrm{t}=12.36, \mathrm{p}<0.001$ ), but lower in neutral pairs (estimate $=-0.044, \mathrm{SE}=0.004, \mathrm{t}=-9.96, \mathrm{p}<0.001$ ). 'All different' and 'goal similar only' pairs were not different from each other (estimate $=0.007, \mathrm{SE}=0.004, \mathrm{t}=$ $1.67, \mathrm{p}=0.09$ ).

To assess the relation between grip and goal priming effects and the perceptual similarity indices in the different goal and grip similarity conditions, Spearman's rank correlations were computed. Table 2 summarizes the results of the analysis. 
APPENDIX 3A: Determination of the random effect structure in mixed-effect models of Experiment 1

Following the recommendation of Barr et al. (2013), we first attempted to fit a model with the maximum random structure, including, for both subjects and items, random intercepts, random slopes for GRIP, GOAL, DURATION, and RESP and random slopes for the 4 possible interactions between GRIP, GOAL, DURATION, and RESP. As expected, the model was overparametrized in regards to the data available and failed to converge to a stable solution (see Bates, Kliegl, Vasisht \& Baayen, 2015; Matuschek, Kliegl, Vasisth, Baayen \& Bates, 2017). Then following the latest recommendations of Bates et al. (2015) we determined the optimal random structure supported by the data. First higher-order random slopes (random slopes reflecting interactions) were removed. Then we looked for possible further reduction of the remaining random effect structure by conducting a principal component analysis on the random terms of the model using the rePCA function from the RePsychLing package version 0.0.4 developed by Bates et al. (2015). The analysis identified 5 components, three of them being sufficient to explain $100 \%$ of variance. The random slope for DURATION was the least representative factor of these three components for subject and was thus removed from the random effect structure of the model. For items, the least representative factor was GRIP and GOAL, the two factors were then removed from the random effect structure of the model. Consequently, the final model included random intercepts, GRIP, GOAL and RESP as random slopes for subject, and random intercepts, DURATION and RESP as random slopes for items.

The following full model was finally considered in Experiment 1 (Bold indicates fixed effects of interest)

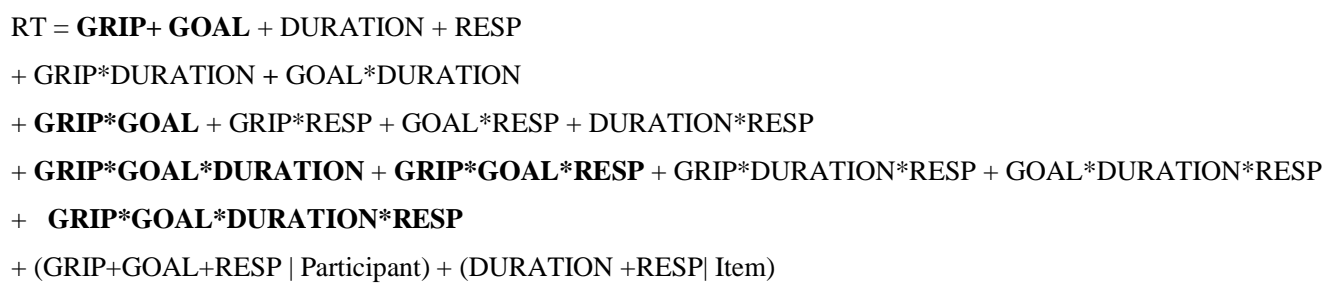

APPENDIX 3B: Determination of random effect structure in mixed effect models of Experiment 2

As for Experiment 1, the maximum random structure was not suited for the data of Experiment 2. Moreover, the full model did not converge with GRIP, GOAL, DURATION, and RESP as random slopes for participants and items. A first principal component analysis on the random terms of the model indicated that the GRIP, GOAL and DURATION for subjects were the least representative factors of the components explaining $100 \%$ of variance and were removed from the analysis. For items, the least representative factors explaining $100 \%$ of variance were GRIP and GOAL and were then removed from the analysis. Thus, the final model included random intercepts and random slopes for RESP for subjects, and random intercepts, RESP and DURATION as random slopes for items.

The following full model was finally considered in Experiment 2 (Bold indicates fixed effects of interest)

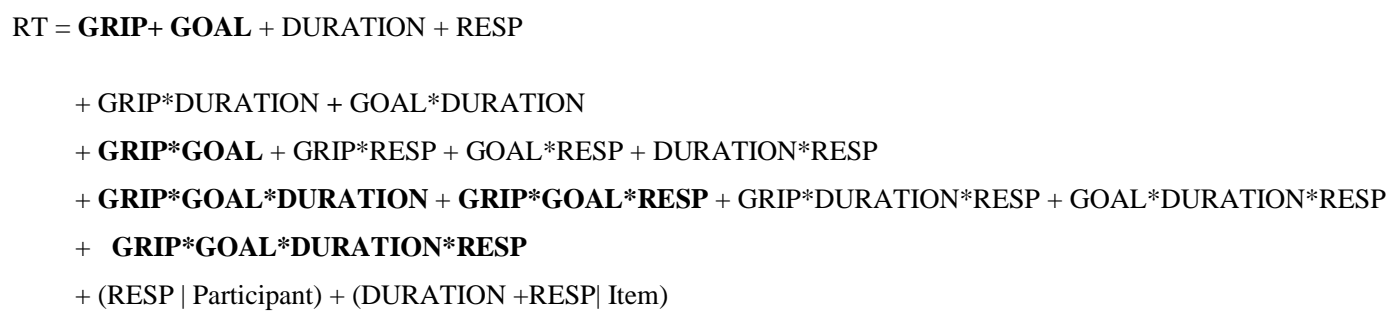


1 Table Captions

2 Table 1 Mean correct reaction times and standard deviations according to prime-target pairs, prime duration (66,

3300 for Experiment 1; 120, 220ms for Experiment 2) and response type in Experiment 1 and 2.

4 Table 2 Spearman's rank correlations between goal and grip similarity priming effects and the corresponding 5 perceptual similarity difference scores 


\section{Figure Captions}

2 Fig. 1 Example of action photographs containing grip and/or goal violations for a given stimulus. In this example, 3 the actress grasps the upright (full and closed) water bottle with a typical clench grip, a correct action regarding 4 the typical function of the object (drink). In the photograph presenting a goal violation, the actress grasps the bottle 5 with the same typical clench grip but the bottle is upside down, preventing her from drinking. In the photograph 6 presenting a grip violation, the actress grasps the bottle with an atypical grip from below that does not prevent her 7 from drinking. The photograph combining grip and goal violations displays the actress grasping the upside-down 8 bottle with the atypical grip. Action photographs are then divided into eight prime-target pairs according grip 9 similarity, goal similarity and response type. An additional neutral prime-target pair was included in Experiment 102 . The FSIM index provides a measure of low-level perceptual similarity between prime and target.

Fig. 2 Individual mean priming effects in milliseconds according to grip similarity, goal similarity, prime duration and response type. Prime durations of 66 and $300 \mathrm{~ms}$ were tested in Experiment 1, and prime durations of 120 and $220 \mathrm{~ms}$ were evaluated in Experiment 2. Boxes represent the interquartile range. Black horizontal lines represent the medians of the distribution. Computed with the pirateplot function of $\mathrm{R}$ ( $\mathrm{R}$ version 3.3.2 ( $\mathrm{R}$ Core Team 2017) and R Studio version 1.0.136.). 


\begin{tabular}{|c|c|c|c|c|c|}
\hline $\begin{array}{c}\text { Response } \\
\text { Type }\end{array}$ & \multicolumn{5}{|c|}{ YES } \\
\hline $\begin{array}{l}\text { Prime- } \\
\text { target } \\
\text { pairs }\end{array}$ & Grip and goal similar & Grip similar only & Goal similar only & All different & $\begin{array}{c}\text { Neutral } \\
\text { (Experiment 2) }\end{array}$ \\
\hline Prime & & & & & \\
\hline \multicolumn{6}{|l|}{ Target } \\
\hline $\begin{array}{c}\text { Grip } \\
\text { similarity } \\
\text { Priming } \\
\text { effect }\end{array}$ & Shared & Shared & Different & Different & NA \\
\hline $\begin{array}{c}\text { Goal } \\
\text { similarity } \\
\text { priming } \\
\text { effect }\end{array}$ & Shared & Different & Shared & Different & NA \\
\hline $\begin{array}{c}\text { FSIM } \\
\text { perceptual } \\
\text { index } \\
\text { mean(SD) }\end{array}$ & 1 & $0,91(+/-0,03)$ & $0,87(+/-0,04)$ & $0,86(+/-0,04)$ & $0,82(+/-0,03)$ \\
\hline $\begin{array}{c}\text { Response } \\
\text { Type }\end{array}$ & \multicolumn{5}{|c|}{ NO } \\
\hline $\begin{array}{c}\text { Prime- } \\
\text { target } \\
\text { pairs }\end{array}$ & All different & Goal similar only & Grip similar only & Grip and goal similar & $\begin{array}{c}\text { Neutral } \\
\text { (Experiment 2) }\end{array}$ \\
\hline Prime & & & & & \\
\hline Target & & & & & \\
\hline $\begin{array}{c}\text { Grip } \\
\text { similarity } \\
\text { Priming } \\
\text { effect }\end{array}$ & Different & Different & Shared & Shared & NA \\
\hline $\begin{array}{c}\text { Goal } \\
\text { similarity } \\
\text { priming } \\
\text { effect }\end{array}$ & Different & Shared & Different & Shared & NA \\
\hline $\begin{array}{c}\text { FSIM } \\
\text { perceptual } \\
\text { index } \\
\text { mean(SD) }\end{array}$ & $0,86(+/-0,04)$ & $0,87(+/-0,04)$ & $0,91(+/-0,03)$ & 1 & $0,81(+/-0,03)$ \\
\hline
\end{tabular}




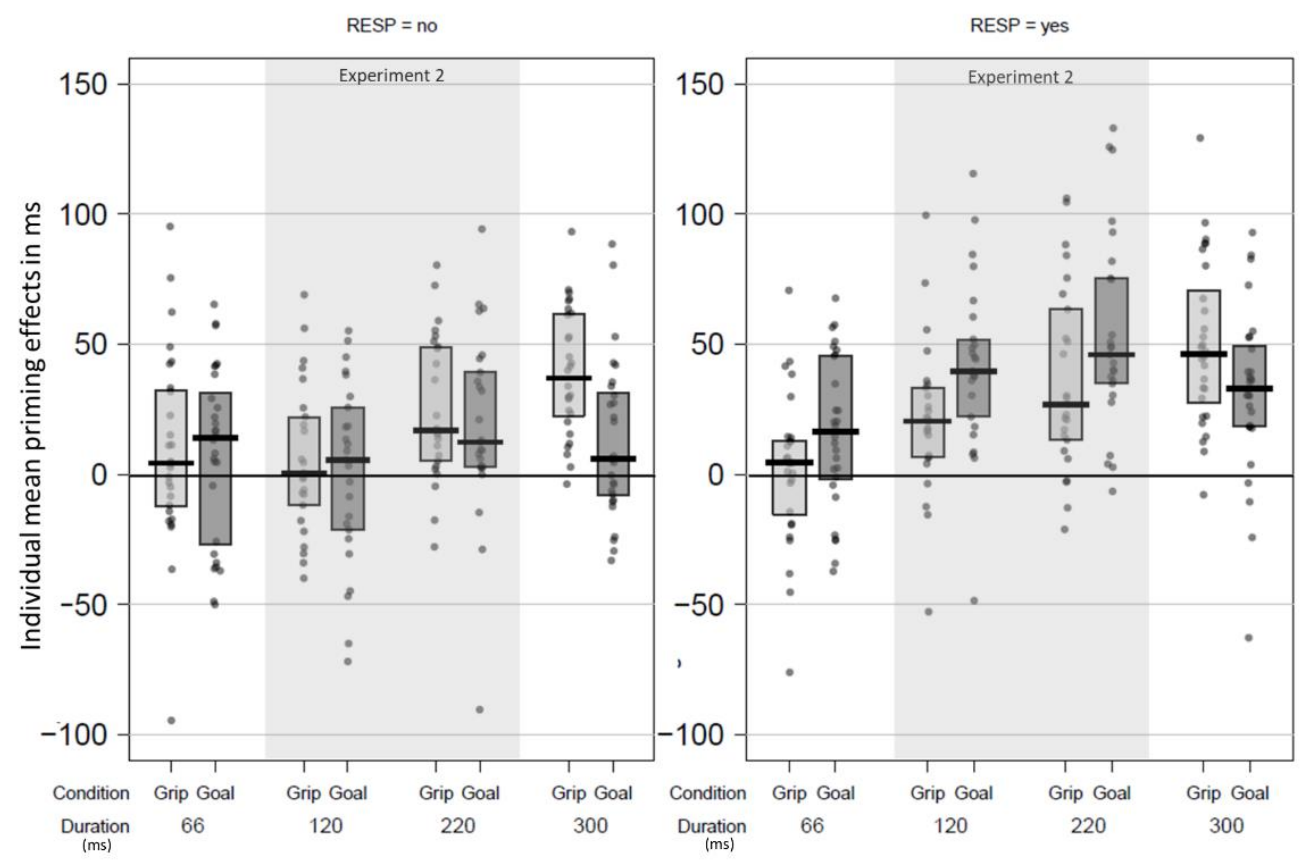

